\title{
Predictors of residual sleepiness in adequately treated obstructive sleep apnoea patients
}

\author{
I. Koutsourelakis, E. Perraki, N.T. Economou, P. Dimitrokalli, E. Vagiakis, \\ C. Roussos and S. Zakynthinos
}

ABSTRACT: Some patients with obstructive sleep apnoea syndrome (OSAS; respiratory distress index (RDI) of $>5$ events $\cdot h^{-1}$ ) experience residual excessive daytime subjective sleepiness (Epworth Sleepiness Scale (ESS) score of $>10$ ), despite adequate use of continuous positive airway pressure (CPAP) therapy. The aim of the present study was to identify clinical and polysomnographic predictors of this sleepiness.

Clinical and polysomnographic variables and ESS score were evaluated in 208 OSAS patients with an ESS score of $>10$ before (initial assessment) and after $\geqslant 6$ months of adequate $\left(\geqslant 4 \mathrm{~h} \cdot \mathrm{day}^{-1}\right)$ CPAP use.

Following CPAP treatment, 114 (55\%) patients showed an abnormal ESS score (>10; CPAP nonresponders), whereas 94 (45\%) showed a normal ESS score ( $<11$; CPAP responders). Of the CPAP responders, none had a history of depression, whereas the converse was true for $38.8 \%$ of CPAP nonresponders. In addition, multivariate logistic regression analysis revealed that the independent predictors of residual excessive daytime sleepiness following CPAP therapy were a history of diabetes and heart disease, and a higher ESS score and lower RDI on initial assessment.

In conclusion, predictors of residual excessive sleepiness in adequately CPAP-treated OSAS were a history of depression, diabetes and heart disease, and a higher ESS score and lower RDI on initial assessment.

KEYWORDS: Depression, diabetes, Epworth Sleepiness Scale, heart disease, obstructive sleep apnoea syndrome, residual excessive sleepiness

ontinuous positive airway pressure (CPAP) is considered the standard treatment of obstructive sleep apnoea syndrome (OSAS) [1]. Results of meta-analyses of randomised controlled trials have suggested that CPAP treatment can reduce subjective sleepiness associated with OSAS, with the greatest benefit found in patients with more severe apnoea and sleepiness $[2,3]$.

However, there are patients who, despite the significant reduction in sleep-disordered breathing with CPAP therapy, continue to complain of excessive daytime sleepiness [4, 5]. Indeed, GuilleminAult and Philip [6] reported that tiredness, fatigue and/or sleepiness persisted in 5\% of OSAS patients despite appropriate treatment. This issue represents an important clinical problem since residual sleepiness in supposedly treated OSAS patients may still lead to significant socioeconomic hardship, including driving and jobrelated accidents [7] and neurocognitive impairment [8]. This is why several clinical trials have tested the efficacy of wake-promoting agents such as modafinil in the treatment of CPAP-resistant daytime sleepiness of OSAS patients [4].

The aetiological factors leading to persistent excessive sleepiness despite ideal CPAP use remain unclear, and only sparse and indirect data have suggested a link with clinical variables, such as obesity [9]. The multifactorial pathophysiology of sleepiness in patients with OSAS before treatment has been well documented [10, 11]. Indeed, the present authors have shown that OSAS, along with common medical comorbid
AFFILIATIONS

Center of Sleep Disorders, Medical School of Athens University, Dept of Critical Care and Pulmonary Services, Evangelismos Hospital, Athens, Greece.

CORRESPONDENCE

I. Koutsourelakis

Medical School of Athens University, Center of Sleep Disorders Evangelismos Hospital 45-47 Ipsilandou Str GR 10675

Athens

Greece

E-mail: ykoutsourelakis@yahoo.gr

Received:

Aug 132008

Accepted after revision: March 232009

First published online: April 82009 
conditions, primarily depression and diabetes, contributes to increased subjective sleepiness of patients suspected of having OSAS [10]. Additionally, polysomnographic variables, such as hypoxaemia or sleep fragmentation, are commonly implicated in the aetiology of daytime sleepiness [11]. Consequently, it is plausible to suggest that persistent excessive daytime sleepiness in effectively treated OSAS patients also results from these clinical and nocturnal factors. Knowledge of such factors prior to CPAP implementation would be valuable for patients, their relatives, physicians and insurance organisations.

Therefore, the aim of the present study was to investigate clinical and polysomnographic determinants of residual excessive daytime subjective sleepiness in CPAP-treated OSAS patients. It was hypothesised that factors that increase daytime sleepiness in OSAS patients before treatment [10] could also predict the persistence of excessive sleepiness when CPAP therapy adequately abolishes sleep-disordered breathing.

\section{METHODS}

\section{Patients}

Patients screened for the current study were subjects who were referred to the Centre of Sleep Disorders of Evangelismos General Hospital (Athens, Greece) between November 2004 and January 2008 for suspected sleep-disordered breathing (fig. 1). Participants were eligible if they met the following two inclusion criteria: 1) a respiratory distress index (RDI) on baseline diagnostic full-night polysomnography of $>5$ events $\cdot h^{-1}$; and 2) excessive daytime sleepiness on initial assessment, manifest in an Epworth Sleepiness Scale (ESS) score of $>10$. Exclusion criteria were: 1) nonacceptance of CPAP therapy; 2) follow-up of $<6$ months; 3) poor compliance with CPAP therapy or insufficient CPAP pressure titration; 4) use of antihistamine or hypnotic medication; 5) coexisting sleep disorders (narcolepsy, idiopathic hypersomnia and periodic limb movement disorder); and 6) shift work. The protocol was approved by the hospital human ethics committee and written informed consent was obtained from all participants.

\section{Study design}

All of the patients underwent three assessments at: baseline, CPAP titration and a follow-up visit $\geqslant 6$ months later. The baseline assessment consisted of overnight polysomnography to confirm the diagnosis of OSAS and self-administration of the ESS, along with clinical screening, as previously described [10]. In brief, patients with documented ischaemic heart disease, hypertension, heart failure or cardiac arrhythmia were classified as having heart disease, and, equally, patients with a documented history of stroke or chronic obstructive pulmonary disease (COPD) were categorised as patients with stroke or COPD, respectively. Additionally, the use of any antidepressant medication prescribed by a psychiatrist and a Center for Epidemiological Studies of Depression (CES-D) score of $>16$ before the initiation of medication were considered to classify a patient in the depression group [12]. In all cases, the patient's psychiatrist was contacted to request the CES-D score and confirm the diagnosis of depression. When such communication was impossible, a sleep physician (I. Koutsourelakis) took the CES-D score from patient recall. Diabetes was defined according to current criteria [13]. Finally, alcohol use was defined as the ingestion of two or more alcoholic drinks daily [14], and smokers were defined as current smokers if any type of tobacco product had been systematically used during the preceding month. All data were ultimately validated by adjudicated chart review by a sleep physician (I. Koutsourelakis).

The second assessment comprised in-laboratory polysomnography for the manual titration of CPAP. A prescription of an appropriate CPAP pressure and a suitable fitting mask were then given to all participants. Subjects were asked to start using a CPAP device that included internal usage (time at pressure) recording capability. All of the patients had followup appointments at 1 and 3 months in the outpatient clinic in order to encourage compliance, unless earlier difficulties with therapy were encountered. Moreover, they were supplied with a telephone number for contacting the medical team in case of questions. None of the patients were informed of the recording capability of the CPAP unit, but they were told to bring it to

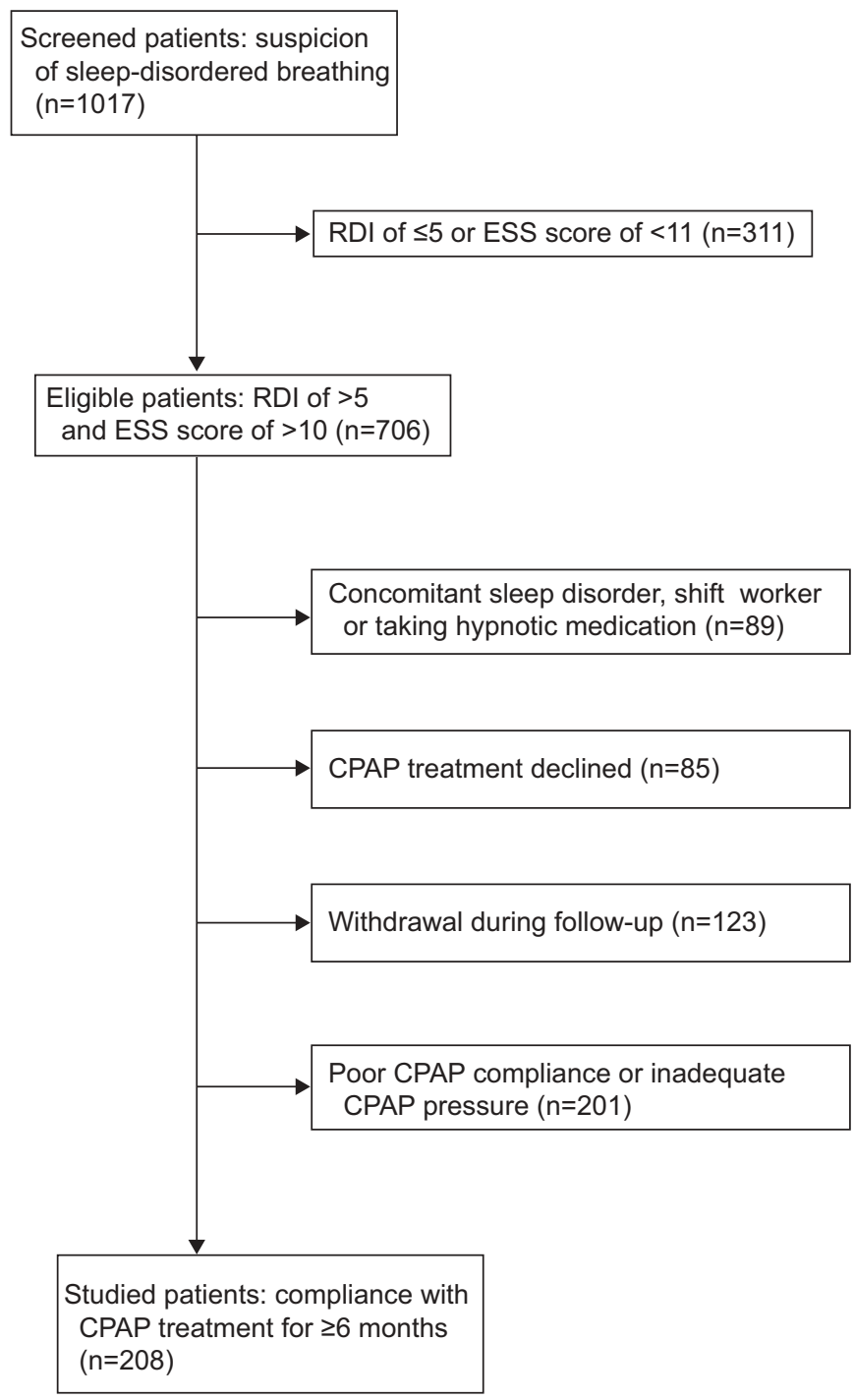

FIGURE 1. Flow chart showing the selection process of the studied patients RDI: respiratory distress index; ESS: Epworth Sleepiness Scale; CPAP: continuous positive airway pressure. 
every follow-up visit. The third assessment took place at a follow-up visit after $\geqslant 6$ months of CPAP use and included a polysomnographic CPAP review study in order to ensure that the CPAP was appropriately titrated. CPAP usage data were then downloaded into a personal computer using proprietary software (Encore pro; Respironics, Inc., Murrysville, PA, USA). Subsequently, all of the patients underwent a review of the clinical screening and self-administered ESS for a second time.

\section{Baseline overnight diagnostic study}

All of the patients underwent overnight polysomnography (EMBLA S7000; Medcare Flaga, Reykjavik, Iceland). In order to determine the stages of sleep, an electroencephalogram (with four channels: C4-A1, C3-A2, O2-A1, and O1-A2), electrooculogram and electromyogram of the submentalis muscle were obtained. The arterial blood oxyhaemoglobin level was recorded using a finger pulse oximeter. Thoracoabdominal excursions were measured qualitatively by respiratory effort sensors (XactTrace; Medcare Flaga) placed over the ribcage and abdomen. Snoring was detected with a vibration snore sensor and body posture with a body position sensor. Airflow was monitored using an oral thermistor and a nasal cannula/ pressure transducer. All variables were recorded using a digital acquisition system (Somnologica 3.3; Medcare Flaga). An experienced technician scored obstructive respiratory events using standard criteria. Thus apnoea was defined as the absence of inspiratory airflow for $>10 \mathrm{~s}$ in the presence of ribcage and abdominal excursions [15], whereas hypopnoea was defined as a $\geqslant 30 \%$ reduction in airflow compared to baseline, lasting $>10 \mathrm{~s}$ and leading to a decrease in haemoglobin saturation of $\geqslant=4 \%$ or an arousal [16]. An arousal was defined as an abrupt shift in electroencephalogram frequency for $\geqslant 3 \mathrm{~s}$ [15]. Respiratory effort-related arousal was defined as a sequence of breaths characterised by increasing respiratory effort leading to an arousal that does not meet criteria for an apnoea or hypopnoea [15]. The number of episodes of apnoeas, hypopnoeas and respiratory effort-related arousals per hour of sleep is referred to as the RDI. OSAS was diagnosed if the RDI was $>5$ events $\cdot h^{-1}[15]$.

\section{CPAP titration study}

Following the baseline diagnostic evaluation, a manual titration of CPAP was performed using a full polysomnographic setting. All of the patients were instructed in the use of CPAP before the night of CPAP titration. The best-fitting mask was chosen, and the patients were familiarised with the CPAP treatment with the help of staff physicians. The optimal CPAP pressure was the minimum pressure needed to abolish snoring and obstructive respiratory events. Adequate titration of CPAP pressure was checked during the polysomnographic CPAP review study that took place at a follow-up assessment.

\section{Apparatus and CPAP compliance}

All of the participants were prescribed a CPAP device (RemStar Auto; Respironics, Inc.) that automatically turned on when the patients breathed into the mask and shut off when the mask was removed. The CPAP device contained a built-in time counter, which recorded the time spent at effective pressure. At the completion of follow-up, objective compliance was assessed using the mean number of hours of daily CPAP use spent at effective pressure. Patients were considered to show good compliance whenever their daily CPAP use during the follow-up period was $\geqslant 4 \mathrm{~h} \cdot \mathrm{day}^{-1}$, and patients were considered to show poor compliance whenever their daily CPAP use was $<4 \mathrm{~h} \cdot$ day $^{-1}$. The cut-off time of $4 \mathrm{~h} \cdot$ day $^{-1}$ has been identified as the threshold above which further improvement in subjective sleepiness is less likely to occur [17]. Nevertheless, since the CPAP time required in order to achieve normal sleepiness should probably be individually determined rather than be generally applicable [17], and the cut-off time of $4 \mathrm{~h} \cdot$ day $^{-1}$ for definition of compliance may be low if a population with long habitual sleep hours is addressed, those patients with daily CPAP use of $\geqslant 5$ and $\geqslant 6 \mathrm{~h} \cdot$ day $^{-1}$ were also evaluated.

\section{Subjective sleepiness and CPAP treatment response}

A subjective tendency for sleepiness was evaluated by means of the validated Greek version [18] of the ESS [19], on initial assessment and at a follow-up assessment $\geqslant 6$ months later. Higher ESS scores indicate a greater subjective tendency for daytime sleepiness. Excessive daytime subjective sleepiness is commonly considered present whenever the ESS score is $>10$ [19], and this limit was also adopted in the present study for the definition of excessive daytime sleepiness. CPAP treatment response was assessed on the basis of post-treatment level of subjective sleepiness, which is regarded as the primary outcome in the clinical management of OSAS [20]. Thus patients who exhibited a normal ESS score $(<11)$ following $\mathrm{CPAP}$ treatment were defined as CPAP responders, whereas those who exhibited an abnormal ESS score $(>10)$ after CPAP treatment, thus demonstrating residual excessive daytime sleepiness, were defined as CPAP nonresponders.

\section{Statistical analysis}

Quantitive data are reported as mean \pm SD. The normality of the data distributions was assessed using the KolmogorovSmirnov test. Differences in the means of quantitative variables between $\mathrm{CPAP}$ responders and $\mathrm{CPAP}$ nonresponders were assessed by unpaired t-test, whereas differences in categorical values were assessed using the Yates-corrected Chi-squared test or Fisher's exact test as appropriate.

Univariate logistic regression analysis was performed in order to determine the contribution of each variable to residual excessive daytime sleepiness following CPAP therapy. Multivariate logistic regression analysis followed in order to identify the variables that were independently associated with residual excessive daytime sleepiness following CPAP therapy. The stepwise procedure was used to select the best logistic regression model, and the goodness of fit of this model was assessed using the Hosmer-Lemeshow test. The independent variables included in the model were those that showed significant difference in the univariate comparison between CPAP responders and CPAP nonresponders; body mass index, stroke and alcohol use were also included in the model, despite lack of significance, because of their potential importance [10, 14]. A p-value of $<0.05$ was considered to indicate significance.

\section{RESULTS}

Of 1,017 patients screened, 311 did not meet the inclusion criteria (109 had an RDI of $\leqslant 5$ events $\cdot h^{-1}$ and 202 had an ESS score of $<11$ ). Of 706 eligible patients, 498 were excluded from analysis (13 had a sleep disorder other than sleep apnoea, 43 
were shift workers, 33 were taking hypnotic medication and 409 fulfilled other exclusion criteria) (fig. 1). The resulting cohort of 208 patients was stratified into CPAP responders (94 patients; $45 \%$ ) and CPAP nonresponders (104 patients; 55\%). The clinical characteristics and polysomnographic data of these two groups of patients determined on baseline assessment are summarised in table 1. Age, ESS score, mean and lowest arterial oxygen saturation, and percentages of diabetes, depression, heart disease and COPD were higher, whereas percentage of males and RDI were lower, in CPAP nonresponders than in CPAP responders. Of 44 patients with depression (all CPAP nonresponders), the CES-D score was documented by their psychiatrist in 36 cases, whereas, in the rest, the CES-D score was taken from the patient's recall. Follow-up evaluation took place after $8.2 \pm 1.2$ months for CPAP responders and $9.1 \pm 1.5$ months for CPAP nonresponders. On follow-up assessment, body mass index remained unchanged for CPAP responders $\left(34.4 \pm 6.7 \mathrm{~kg} \cdot \mathrm{m}^{-2}\right)$ and CPAP nonresponders $\left(34.3 \pm 8.1 \mathrm{~kg} \cdot \mathrm{m}^{-2}\right)$. Additionally, treatment for various diseases, e.g. diabetes or heart disease, did not change substantially during follow-up. CPAP responders exhibited a mean decrease in ESS score of $8.4 \pm 2.7$ after CPAP treatment, whereas CPAP nonresponders showed a mean decrease in ESS score of $2.4 \pm 2.4$ after CPAP treatment $(\mathrm{p}<0.001)$. Additionally, objective compliance was not significantly different between the two groups: $6.1 \pm 1.0 \mathrm{~h} \cdot$ night $^{-1}$ for CPAP responders (mean prescribed CPAP pressure of $8.4 \pm 1.2 \mathrm{cmH}_{2} \mathrm{O}$ ); and $5.9 \pm 0.9 \mathrm{~h} \cdot$ night $^{-1}$ for CPAP nonresponders (mean prescribed CPAP pressure of $6.7 \pm$ $1.6 \mathrm{cmH}_{2} \mathrm{O}$ ). Finally, the RDI on follow-up CPAP review polysomnography was within normal limits $\left(2.2 \pm 1.1\right.$ events $\cdot h^{-1}$ for CPAP responders and $3.1 \pm 0.9$ events $\cdot h^{-1}$ for CPAP nonresponders).

Multivariate logistic regression analysis was performed in order to identify the independent predictors of residual excessive daytime sleepiness following CPAP therapy. Demographic, clinical and polysomnographic parameters determined on baseline assessment that showed significant difference in the univariate comparison between CPAP responders and CPAP nonresponders (and also body mass index, stroke and alcohol use) (table 1) were entered into a forward stepwise logistic regression model. Since depression offered near-complete discrimination between CPAP responders and CPAP nonresponders $(0 \%$ of $\mathrm{CPAP}$ responders and $38.6 \%$ of CPAP nonresponders had a history of depression), it was not included in the logistic regression model because maximum likelihood estimation could not be achieved. By examining the effect of the other variables, multivariate logistic regression analysis revealed that the independent predictors of residual subjective sleepiness following CPAP therapy were the presence of diabetes, heart disease, a higher ESS score and a lower RDI on baseline assessment (table 2). The HosmerLemeshow test indicated that the fit of the model was good $(p=0.68)$. The backward procedure gave identical results.

By performing ad hoc analysis, it was found that, if the cut-off CPAP use for good compliance was set at $5 \mathrm{~h} \cdot \mathrm{day}^{-1}$, the resulting cohort included 125 patients divided into $89(71 \%)$ CPAP responders and 36 (29\%) CPAP nonresponders, whereas, if the cut-off time for good compliance was set at $6 \mathrm{~h} \cdot$ day $^{-1}$, the cohort consisted of 94 patients, stratified into 69 (73\%) CPAP responders and 25 (27\%) CPAP nonresponders.

\begin{tabular}{|c|c|c|}
\hline \multirow[t]{2}{*}{ TABLE 1} & \multicolumn{2}{|c|}{$\begin{array}{l}\text { Demographic, clinical and polysomnographic } \\
\text { features of obstructive sleep apnoea syndrome } \\
\text { patients with excessive daytime sleepiness }{ }^{\#} \text { on } \\
\text { baseline assessment }\end{array}$} \\
\hline & CPAP responders & CPAP nonresponders \\
\hline Subjects $\mathrm{n}$ & 94 & 114 \\
\hline Age yrs & $50.7 \pm 12.2 ; 51$ (27-78) & $57.8 \pm 12.5 ; 59(20-81)^{\star * *}$ \\
\hline $\mathrm{BMI} \mathbf{k g} \cdot \mathrm{m}^{-2}$ & $34.2 \pm 7.1 ; 32(24-60)$ & $33.9 \pm 8.3 ; 32(20-72)$ \\
\hline Males & $86.2(79.2-93.2)$ & $73.7(65.6-81.8)^{*}$ \\
\hline RDI events $\cdot h^{-1}$ & $59.4 \pm 33.7 ; 60.7(6.4-133.6)$ & $44.5 \pm 28.2 ; 35.2(6.5-121.2)^{\star \star}$ \\
\hline ESS score & $14.0 \pm 3.0 ; 13(11-23)$ & $16.2 \pm 3.0 ; 16(11-23)^{\star *}$ \\
\hline Diabetes & $6.4(1.4-11.3)$ & $42.1(33.1-51.2)^{\star \star \star}$ \\
\hline Depression & 0 & $38.6(29.7-47.5)^{\star \star \star}$ \\
\hline Hypothyroidism & $5.3(0.8-9.9)$ & $11.5(5.6-17.2)$ \\
\hline Alcohol use & $17.0(9.4-24.6)$ & $14.9(8.4-21.5)$ \\
\hline Heart disease & $40.4(30.5-50.4)$ & $67.5(58.9-76.1)^{\star \star \star *}$ \\
\hline COPD & $7.4(2.1-12.8)$ & $19.3(12.1-26.5)^{\star}$ \\
\hline Stroke & $11.7(5.2-18.2)$ & $13.2(7.0-19.4)$ \\
\hline Smokers & $39.4(29.5-49.2)$ & $42.1(33.1-51.1)$ \\
\hline REM/TST \% & $11.6 \pm 7.6 ; 10.3(0-38.3)$ & $10.2 \pm 7.3 ; 9.6(0-30.6)$ \\
\hline S1/TST \% & $5.4 \pm 6.2 ; 4.0(1.1-37.9)$ & $5.4 \pm 5.0 ; 3.6(0.8-25.1)$ \\
\hline S2/TST \% & $81.7 \pm 17.5 ; 84.3(1.7-99.0)$ & $80.8 \pm 13.3 ; 82.5(12.1-98.5)$ \\
\hline SWS/TST \% & $2.4 \pm 8.6 ; 0(0-56.9)$ & $3.3 \pm 10.4 ; 0(0-63.5)$ \\
\hline Mean $\mathrm{Sa}_{1} \mathrm{O}_{2} \%$ & $89.4 \pm 6.5 ; 91.1$ (64-96.8) & $91.3 \pm 4.3 ; 92.3(73.3-97.6)^{*}$ \\
\hline Lowest $\mathrm{Sa}, \mathrm{O}_{2} \%$ & $71.2 \pm 11.1 ; 72(46-91)$ & $75.4 \pm 10.1 ; 78(50-91)^{\star *}$ \\
\hline
\end{tabular}

Patients who exhibited a normal Epworth Sleepiness Scale (ESS) score $(<11)$ following continuous positive airway pressure (CPAP) treatment (mean CPAP use of $\geqslant 4 \mathrm{~h} \cdot$ day $^{-1}$ during follow-up period) were defined as CPAP responders, whereas those who exhibited an abnormal ESS score $(>10)$ after CPAP treatment, thus demonstrating residual excessive daytime sleepiness, were defined as CPAP nonresponders. Continuous data are presented as mean \pm SD with median (interquartile range), whereas categorical data are presented as percentage (95\% confidence interval). BMI: body mass index; RDI: respiratory distress index; COPD: chronic obstructive pulmonary disease; REM: rapid eye movement sleep; TST: total sleep time; S1: stage 1 sleep; S2: stage 2 sleep; SWS: slow-wave sleep; $\mathrm{Sa}_{1} \mathrm{O}_{2}$ : arterial oxygen saturation. ${ }^{\#}$ : ESS score of $>10$. *: $p<0.05 ; * *: p<0.01 ; * \star *: p<0.001$ versus CPAP responders.

The clinical characteristics and polysomnographic data of these two cohorts of patients determined on baseline assessment are summarised in tables $1 S$ and $2 S$ of the online supplementary material, respectively. For most variables, the results were identical to those of the original cohort (table 1), since age and percentages of diabetes, depression, heart disease and COPD were higher, whereas RDI and percentage of males were lower, in CPAP nonresponders than in CPAP responders. Additionally, by applying univariate and multivariate logistic regression models (table 35 of the online supplementary material), the results obtained did not differ between the three cohorts. Indeed, the odds ratios and 95\% confidence intervals that describe the magnitude of the effects were reasonably similar. Minimal changes that were observed in multivariate logistic models, e.g. the effect of heart disease was slightly weaker in the two cohorts derived from ad hoc analysis than in the original cohort and nonsignificant, could be explained by sample size reduction and the consequent power loss. 


\begin{tabular}{|c|c|c|c|}
\hline \multirow[t]{2}{*}{ TABLE 2} & \multicolumn{3}{|c|}{$\begin{array}{l}\text { ORs of variables independently associated with } \\
\text { residual excessive daytime sleepiness following } \\
\text { continuous positive airway pressure therapy }\end{array}$} \\
\hline & & OR $(95 \% \mathrm{Cl})$ & p-value \\
\hline \multicolumn{2}{|l|}{ Diabet } & $6.87(2.50-18.91)$ & $<0.001$ \\
\hline \multicolumn{2}{|c|}{ Heart disease $(0=$ no; $1=$ yes $)$} & $2.94(1.41-6.15)$ & 0.004 \\
\hline \multicolumn{2}{|c|}{ Epworth Sleepiness Scale score } & $1.31(1.15-1.49)$ & $<0.001$ \\
\hline \multicolumn{2}{|c|}{ Respiratory distress index } & $0.97(0.96-0.98)$ & $<0.001$ \\
\hline \multicolumn{4}{|c|}{$\begin{array}{l}\text { All of the variables were determined on baseline assessment. Results were } \\
\text { calculated using stepwise forward multivariate logistic regression analysis. } \\
\text { Variables that showed significant difference in the univariate comparison } \\
\text { between CPAP responders and CPAP nonresponders (and also body mass } \\
\text { index, stroke and alcohol use) were entered into the model. Depression was not } \\
\text { included in the model because it offered near-complete discrimination between } \\
\text { CPAP responders and CPAP nonresponders and maximum likelihood estima- } \\
\text { tion was not achieved. Among patients, the odds of having residual excessive } \\
\text { daytime sleepiness following CPAP therapy increased with the presence of } \\
\text { diabetes, heart disease and a higher Epworth Sleepiness Scale score and a } \\
\text { lower respiratory distress index on baseline assessment. }\end{array}$} \\
\hline
\end{tabular}

\section{DISCUSSION}

The present study demonstrated that, in adequately CPAPtreated OSAS patients, residual excessive daytime sleepiness following effective CPAP therapy is independently associated with a history of depression, diabetes and heart disease, and the presence of a higher ESS score and lower RDI on initial assessment.

The literature reports a variety of clinical and polysomnographic factors, in addition to apnoeas, that are associated with increased subjective sleepiness of OSAS patients [10, 11, 14]. However, none of these studies directly examined the contribution of these factors to the subjective sleepiness of OSAS patients who remain subjectively somnolent despite implementation of CPAP therapy and the ensuing abolition of sleep-disordered breathing. Moreover, a number of previous studies [5, 9, 17, 21, 22] have highlighted residual excessive sleepiness of OSAS patients following CPAP implementation, but none have examined the determinants of this sleepiness beyond factors related to sufficient CPAP therapy [5, 17, 21, 22]. The present study adds to the current literature by employing strict exclusion criteria, which aimed to convincingly eliminate the effect of apnoeas on subjective sleepiness and thus render the contribution of the rest of factors more apparent.

Daytime sleepiness refractory to adherent use of CPAP is of paramount importance for patients with OSAS and their relatives and physicians, but also for insurance companies, since even modest levels of sleepiness may have a substantial negative effect on daytime functioning, quality of life and safety $[7,8]$. Despite its significance, the prevalence of residual excessive daytime sleepiness in patients with OSAS who are undergoing CPAP treatment remains unknown. The present study was not designed to evaluate the prevalence of this condition, as it enrolled only patients who used adequately titrated CPAP for $>4 \mathrm{~h} \cdot \mathrm{day}^{-1}$ for $\geqslant 6$ months. Indeed, of 706
OSAS patients who met the inclusion criteria, 498 were excluded from further analysis for various reasons (fig. 1). Therefore, due to selection bias associated with the high percentage of excluded patients, the present study could not evaluate the residual excessive daytime sleepiness in unselected CPAP-treated patients. Literature reports contain various percentages of residual excessive daytime sleepiness in treated OSAS patients. WEAVER et al. [17] demonstrated that subjective sleepiness persisted in 34\% of patients despite using CPAP, whereas GUILLEMINAULT and PHILIP [6] reported that 5\% of patients with sleep-disordered breathing still complained of daytime tiredness, fatigue and/or sleepiness despite various treatment strategies (CPAP, oral appliances and surgery) [6]. In the present study, the percentage of patients with remaining subjective sleepiness following mean CPAP use of $\geqslant 4 \mathrm{~h} \cdot$ day $^{-1}$ was high $(55 \%)$. However, if the time for definition of good compliance were set at $\geqslant 5$ or $\geqslant 6 \mathrm{~h} \cdot \mathrm{day}^{-1}$, subjective sleepiness persisted in only 29 and $27 \%$ of patients, respectively (tables $1 S$ and $2 \mathrm{~S}$ of the online supplementary material), percentages that are similar to that (34\%) previously reported [17]. Indeed, although WEAVER et al. [17] considered $4 \mathrm{~h} \cdot$ day $^{-1}$ as the cut-off time for CPAP good compliance, they pointed out that the time genuinely needed for CPAP in order to achieve normal levels of sleepiness is likely to be individually determined rather than generally applicable. Thus it appears that, in a population with long habitual sleep hours, as was probably the present one, the cut-off time of $4 \mathrm{~h} \cdot$ day $^{-1}$ for definition of CPAP compliance may be low, and cut-off times of 5 or $6 \mathrm{~h} \cdot$ day $^{-1}$ might better reflect good CPAP compliance.

Interestingly, nearly $40 \%$ of CPAP nonresponders had a history of depression, whereas none of the CPAP responders had such a history (table 1), suggesting that residual excessive sleepiness in adequately CPAP-treated OSAS patients is associated with the presence of depression. Accordingly, it appears that sleep physicians should not always anticipate complete resolution of subjective sleepiness in OSAS patients with depression following implementation of CPAP. Importantly, the overlap of OSAS and depression is not unusual as SCHWARTZ et al. [23] documented that 39\% of patients with obstructive sleep apnoea referred to a sleep unit were receiving antidepressant treatment. The possible causal pathways linking persistent sleepiness with depression in CPAP-treated OSAS patients have been suggested by SANTAMARIA et al. [24], who primarily highlighted the disturbances in pathways involved in the regulation of sleep and wakefulness induced by depression per se, as well as the sedative effect of antidepressant treatment and poor compliance with CPAP therapy.

Diabetes also proved to be a significant determinant of CPAPresistant excessive daytime sleepiness. The association between diabetes and daytime sleepiness is not novel and has been documented in population-based studies, in which diabetes was more prevalent in patients with excessive daytime sleepiness [14]. Although epidemiological studies can only establish an association rather than a causal pathway, diminished cerebral use of glucose and increased sympathetic tone have been suggested to link subjective sleepiness with impaired glucose tolerance [25]. Combining these data, it is plausible to suggest that diabetes should always be ranked in 
the differential diagnosis of persistent daytime sleepiness despite CPAP therapy in OSAS patients.

A history of heart disease was found to predict the occurrence of residual excessive sleepiness in CPAP-treated OSAS patients. This finding is consistent with previous reports that documented an association of daytime sleepiness with the use of medication for congestive heart failure [26] and the occurrence of hypertension [27]. Additionally, it has been suggested that, in patients with heart disease, daytime sleepiness derives from disturbed sleep continuity and poor sleep quality [26].

Finally, an increased ESS score and lower RDI on initial assessment proved to be determinants of residual excessive daytime sleepiness. In other words, OSAS patients with more subjective sleepiness and less severe apnoea were more likely to continue to experience subjective sleepiness following optimal CPAP treatment. This finding seems partially contradictory to the results of a meta-analysis of placebo-controlled trials demonstrating that the more severe the apnoea and sleepiness the greater the benefit obtained in terms of resolution of subjective sleepiness [2]. Indeed, although the present result concerning apnoea severity is in line with that of the meta-analysis [2], the present finding regarding subjective sleepiness severity seems divergent. However, this discrepancy could be attributed to the patient cohort of the current study, which did not include patients with a normal ESS score on initial assessment and patients with OSAS who were not compliant with CPAP use, as well as to the dichotomous patient stratification among $\mathrm{CPAP}$ responders and CPAP nonresponders.

Inadequate CPAP use and the presence of residual respiratory events due to insufficient CPAP titration have been considered leading candidates for causing residual daytime sleepiness in OSAS patients $[21,22]$. Despite the fact that $4 \mathrm{~h} \cdot \mathrm{day}^{-1}$ of CPAP use is the threshold above which further improvement in daytime sleepiness is less likely [17], it could be argued that inadequate mean daily CPAP use might have contributed to residual sleepiness in some patients in the present study, since the cut-off time of $4 \mathrm{~h} \cdot \mathrm{day}^{-1}$ adopted for definition of CPAP compliance may be low in a population with long habitual sleep hours. This possibility cannot be excluded, since during the ad hoc analysis, it was found that the percentage of patients with remaining subjective sleepiness decreased from 55 to 29 and $27 \%$ if the time set for definition of good CPAP compliance were $\geqslant 5$ or $\geqslant 6 \mathrm{~h} \cdot$ day $^{-1}$, respectively. This was due to the fact that more patients with mean CPAP use of $\geqslant 4$ and $<5 \mathrm{~h} \cdot$ day $^{-1}$ were CPAP nonresponders than CPAP responders (78 versus five patients; the correspondent numbers for patients with mean CPAP use of $\geqslant 5$ and $<6 \mathrm{~h} \cdot$ day $^{-1}$ were 11 versus 20 patients (table 1 and tables $1 S$ and $2 S$ of the online supplementary material)), raising the suspicion that several patients with mean CPAP use of $\geqslant 4 \mathrm{~h} \cdot$ day $^{-1}$ might have persistent subjective sleepiness because of an inadequate duration of daily CPAP use. Nevertheless, independent predictors of residual excessive daytime sleepiness following CPAP therapy (i.e. a history of depression, diabetes and heart disease, and a higher ESS score and lower RDI on initial assessment) were exactly the same regardless of whether the cut-off time for CPAP compliance were set at 4,5 or $6 \mathrm{~h} \cdot$ day $^{-1}$, indicating that the results of the present study stand, despite the definition of CPAP compliance. Given that 14 days of CPAP use are sufficient for patients to resume regular alertness [22], the minimum 6-month follow-up of the present study makes it unlikely that residual subjective sleepiness observed in the present study sample could be explained by inadequate duration of CPAP use. Additionally, the contribution of arousals and residual respiratory events can also safely be excluded, since all patients underwent a follow-up CPAP titration review study in order to ensure adequate titration of CPAP pressure. Other potential sources of residual daytime sleepiness include intermittent hypoxaemia [11, 28, 29], age $[14]$, obesity $[9,10,14]$ and a history of stroke [10], COPD [10, 30] and alcohol use [10, 14]. All of these factors failed to be identified as independent determinants of residual daytime sleepiness following CPAP therapy in the present study, and their role in residual sleepiness in this clinical condition should rather be considered minimal or negligible.

Some methodological issues should be acknowledged and warrant consideration in the present study. First, the mean nightly duration of CPAP use in the present study is longer than in previous studies [17]. This is because the aim was to investigate only patients with objective compliance to CPAP of $\geqslant 4 \mathrm{~h} \cdot \mathrm{day}^{-1}$. Thus it does not represent an estimate of general CPAP use. Secondly, sleepiness was assessed using the ESS, a simple self-administered questionnaire that quantifies subjective sleep propensity. However, it remains unclear whether the results would have persisted if an objective measure of sleepiness, such as the multiple sleep latency test, were employed. However, methods used in routine clinical practice and recommended by the American Academy of Sleep Medicine [15] were used.

In conclusion, the results of the present study suggest that a history of depression, diabetes and heart disease, and the presence of a higher ESS score and lower RDI on initial assessment can predict those OSAS patients who may experience excessive daytime sleepiness even following adequate $\left(\geqslant 4 \mathrm{~h} \cdot\right.$ day $\left.^{-1}\right)$ CPAP use. Clinicians should consider these factors in order to sufficiently anticipate the resolution or persistence of excessive daytime sleepiness in OSAS patients.

\section{SUPPORT STATEMENT}

The present study was funded by the Thorax Foundation (Athens, Greece).

\section{STATEMENT OF INTEREST}

None declared.

\section{REFERENCES}

1 Kushida C, Littner M, Hirshkowitz M, et al. Practice parameters for the use of continuous and bilevel positive airway pressure devices to treat adult patients with sleep-related breathing disorders. Sleep 2006; 29: 375-380.

2 Patel S, White D, Malhotra A, et al. Continuous positive airway pressure therapy for treating sleepiness in a diverse population with obstructive sleep apnea. Results of a meta-analysis. Arch Intern Med 2003; 163: 565-571.

3 Marshall NS, Barnes M, Travier N, et al. Continuous positive airway pressure reduces daytime sleepiness in mild to moderate 
obstructive sleep apnoea: a meta-analysis. Thorax 2006; 61: 430-434.

4 Kingshott R, Vennelle M, Coleman E, et al. Randomized, doubleblind, placebo-controlled crossover trial of modafinil in the treatment of residual excessive daytime sleepiness in the sleep apnea/hypopnea syndrome. Am J Respir Crit Care Med 2001; 163: 918-923.

5 Bedard MA, Montplaisir J, Malo J, et al. Persistent neuropsychological deficits and vigilance impairment in sleep apnea syndrome after treatment with continuous positive airways pressure (CPAP). J Clin Exp Neuropsychol 1993; 15: 330-341.

6 Guilleminault C, Philip P. Tiredness and somnolence despite initial treatment of obstructive sleep apnea syndrome (what to do when an OSAS patient stays hypersomnolent despite treatment). Sleep 1996; 19: Suppl., S117-S122.

7 George C. Sleep apnea, alertness, and motor vehicle crashes. Am J Respir Crit Care Med 2007; 176: 954-956.

8 Nowak M, Kornhuber J, Meyrer R. Daytime impairment and neurodegeneration in OSAS. Sleep 2006; 29: 1521-1530.

9 Morisson F, Decary A, Petit D, et al. Daytime sleepiness and EEG spectral analysis in apneic patients before and after treatment with continuous positive airway pressure. Chest 2001; 119: 45-52.

10 Koutsourelakis I, Perraki E, Bonakis A, et al. Determinants of subjective sleepiness in suspected obstructive sleep apnoea. J Sleep Res 2008; 17: 437-443.

11 Punjabi N, O'Hearn D, Neubauer D, et al. Modeling hypersomnolence in sleep-disordered breathing: a novel approach using survival analysis. Am J Respir Crit Care Med 1999; 159: 1703-1709.

12 Shafer AB. Meta-analysis of the factor structures of four depression questionnaires: Beck, CES-D, Hamilton, and Zung. J Clin Psychol 2006; 62: 123-146.

13 American Diabetes Association. Diagnosis and classification of diabetes mellitus. Diabetes Care 2005; 28: Suppl. 1, S37-S42.

14 Bixler E, Vgontzas A, Lin H, et al. Excessive daytime sleepiness in a general population sample: the role of sleep apnea, age, obesity, diabetes and depression. J Clin Endocrinol Metab 2005; 90: 45104515.

15 American Academy of Sleep Medicine Task Force, Sleep-related breathing disorders in adults: recommendations for syndrome definition and measurement techniques in clinical research. Sleep 1999; 22: 667-689.

16 Meoli A, Casey K, Clark R, et al. Hypopnea in sleep-disordered breathing in adults. Sleep 2001; 24: 469-470.
17 Weaver T, Maislin G, Dinges D, et al. Relationship between hours of CPAP use and achieving normal levels of sleepiness and daily functioning. Sleep 2007; 30: 711-719.

18 Tsara V, Serasli E, Amfilochiou A, et al. Greek version of the Epworth Sleepiness Scale. Sleep Breath 2004; 8: 91-95.

19 Johns M. A new method for measuring sleepiness: the Epworth Sleepiness Scale. Sleep 1991; 12: 540-545.

20 Vaughn B, D'Cruz O. Cardinal manifestations of sleep disorders In: Kryger M, Roth T, Dement W, eds. Principles and Practice of Sleep Medicine. 4th Edn. Philadelphia, Elsevier Saunders, 2005; pp. 594-601.

21 Dinges D, Weaver T. Effects of modafinil on sustained attention performance and quality of life in OSA patients with residual sleepiness while being treated with nCPAP. Sleep Med 2003; 4: 393-402.

22 Lamphere J, Roehrs T, Wittig R, et al. Recovery of alertness after CPAP in apnea. Chest 1989; 96: 1364-1367.

23 Schwartz D, Kohler W, Karatinos G. Symptoms of depression in individuals with obstructive sleep apnea may be amenable to treatment with continuous positive airway pressure. Chest 2005; 128: 1304-1309.

24 Santamaria J, Iranzo A, Ma Montserrat J, et al. Persistent sleepiness in CPAP treated obstructive sleep apnea patients: evaluation and treatment. Sleep Med Rev 2007; 11: 195-207.

25 Barceló A, Barbé F, de la Peña M, et al. Insulin resistance and daytime sleepiness in patients with sleep apnoea. Thorax 2008; 63: 946-950.

26 Redeker N, Stein S. Characteristics of sleep in patients with stable heart failure versus a comparison group. Heart Lung 2006; 35: 252-261.

27 Whitney C, Enright P, Newman A, et al. Correlates of daytime sleepiness in 4578 elderly patients: the Cardiovascular Health Study. Sleep 1998; 21: 27-36.

28 Gozal D, Kheirandish L. Sleepiness and neurodegeneration in sleep-disordered breathing: convergence of signaling cascades. Am J Respir Crit Care Med 2005; 171: 1325-1327.

29 Veasey S, Davis C, Fenik P. Long-term intermittent hypoxia in mice: protracted hypersomnolence with oxidative injury to sleepwake brain regions. Sleep 2004; 27: 194-201.

30 Kapur V, Baldwin C, Resnick H, et al. Sleepiness in patients with moderate to severe sleep-disordered breathing. Sleep 2005; 28: 472-477. 